\begin{tabular}{|l|l|l|l|}
\hline Eiszeitalter und Gegenwart & Band 19 & Seite 190-196 & Öhringen/Württ., 31. Oktober 1968 \\
\hline
\end{tabular}

\title{
Zum Alter des Plaggeneschs
}

\author{
Von E. Mückenhausen, H. W. Scharpenseel und F. Pietig, Bonn
}

Mit 1 Abbildung und 1 Tabelle

\begin{abstract}
$\mathrm{Z}$ us a mmenf assung. Der Plaggenesch entstand durch jahrhundertelange Düngung mit Plaggendung, der hauptsächlich aus Plaggen, d.h. flach abgehackten Heide- oder Grasstücken, und Stalldung bestand. Dadurch wurde der $A_{p}$-Horizont über dem ursprünglichen Bodentyp immer mächtiger bis zu $80 \mathrm{~cm}$ und mehr, und damit entstand der anthropogene Bodentyp „Plaggenesch". Entstehung, Aufbau, Eigenschaften und Verbreitung des Plaggeneschs werden beschrieben. Es wird geschildert, wie man das Alter, den Beginn der Plaggendüngung feststellen kann. Hinweise geben Ortsnamen, die Geschichte der Ackerkultur sowie Scherben und andere Funde. Eine direkte Altersbestimmung erlaubt die ${ }^{14} \mathrm{C}$-Methode, indessen ist sie mit Fehlern behaftet, da die organische Masse der Plaggenesche nicht einheitlich ist, d. h. ein verschiedenes Alter haben kann. Es wird daher nur ein Mittelwert erzielt. Unter Beachtung aller Fehlerquellen wird auf Grund der bisher gewonnenen ${ }^{14} \mathrm{C}-$ Werte das Alter der Plaggenesche, d. h. der Beginn der Plaggendüngung, mit etwa 800-1200 Jahren angenommen. Dieses Alter stimmt mit den vorher auf anderem Weg gewonnenen Altersdatierungen einigermaßen überein.

$\mathrm{S} \mathrm{u} \mathrm{m} \mathrm{m} \mathrm{a} \mathrm{r} \mathrm{y.} \mathrm{The} \mathrm{"Plaggenesch“} \mathrm{(Plaggept)} \mathrm{has} \mathrm{been} \mathrm{formed} \mathrm{by} \mathrm{century} \mathrm{long} \mathrm{broad-casting}$ of plaggen-manure, that consists mainly of plaggen, i. e. flatly cut bits and pieces of heath and pasture grass, mixed with stable manure. Thus, the $A_{p}$-horizon beyond the original soil type was growing up to a thickness of $80 \mathrm{~cm}$ and more, creating the anthopogenic soil type „Plaggenesch". Origin, formation, properties and distribution of "Plaggenesch" are described. Further it is dealt with approaches to determine the age, the beginning of plaggen manuring. Names of villages, agraric history, potsherds and other findings give testimony and suggestions. A direct age determination is possible by the ${ }^{14} \mathrm{C}$-method, though a certain error latitude is invitable due to age variations within the organic masses of the "Plaggenesch" themselves. A mean age value is therefore determined. Under recognition of all sources of error the age of "Plaggenesch", i. e. the beginning of plaggen manuring, is assumed to be $800-1200$ years. This age agrees sufficiently well with estimates obtained by other dating methods.
\end{abstract}

Der Plaggenesch ist ein anthropogener Bodentyp des nordwesteuropäischen Festlandes; die meisten Flächen liegen in Nordwestdeutschland. Zunächst wurde dieser Bodentyp „Eschboden“ genannt, weil er hauptsächlich auf dem Esch, der etwas erhöht liegenden Feldflur im nordwestdeutschen Raum vorkommt. Da er indessen auch in niedrigeren Lagen, näher dem Grundwasser, nämlich über dem Gley, auftritt, hat man ihm den Namen „Plaggenboden“ gegeben. Die Regeln der Bodensystematik schließen das Wort „boden“ möglichst aus, deshalb wurde die Bezeichnung "Plaggenesch" eingeführt, ungeachtet der Tatsache, daß dieser Bodentyp nicht ausschließlich „auf dem Esch“ liegt.

Die Literatur über den Plaggenesch ist nicht umfangreich, 1962 hat MückeNHAUSEN die bis dahin erschienene fast vollständig zusammengestellt; es fehlte eine Arbeit von Niemeier (1955). Danach erschien 1962 eine weitere Arbeit von Fastabend und v. Rau$\mathrm{PACH}$, in der Altersangaben über den Plaggenesch mitgeteilt werden. WoHLRAB und LANGNER berichteten 1965 über den Wasserhaushalt der Plaggenesche.

\section{Die Entstehung und der Aufbau des Plaggeneschs}

Der Bodentyp „Plaggenesch“ ist im Fachkreis der Bodenkundler zwar bekannt, indessen vielleicht nicht eingehend jedem des Leserkreises dieses Jahrbuches. Darum sollen seine Entstehung und sein Aufbau kurz dargestellt werden.

Der Plaggenesch wurde vom Menschen geschaffen, und zwar im Bestreben einer grundlegenden Bodenverbesserung. Viele Böden des nordwesteuropäischen Festlandes sind arme Sandböden. Zur Verbesserung dieser Böden erschien schon den Ackerbauern früherer Zeit eine Steigerung der Wasserkapazität und eine Erhöhung des Gehaltes an Pflanzennährstoffen notwendig. Beides wurde angestrebt, indem ein Dünger aus Plaggen, auch Soden 
genannt, und Stalldung hergestellt und auf den Acker gebracht wurde. Überwiegend wurden Heideplaggen, seltener Grasplaggen und Waldplaggen verwendet. Die flach abgehackten Plaggen wurden meistens als Einstreu, hauptsächlich im Schafstall, verwendet, teils wurden sie auch mit Stalldung kompostiert. Bei der Kompostierung sind auch andere organische Stoffe, teils auch mineralische Massen (organische Abfälle, Grabenaushub, Erde u. a.) mitverwendet worden. Die Plaggen bestehen naturgemäß aus organischer und mineralischer Substanz; soweit es sich um Heideplaggen handelt, war das Material sehr stark sauer, so daß ein langsamer Abbau der organischen Masse des Plaggendungs stattfand. Die Düngung mit der mineralreichen Masse hatte zur Folge, daß sich im Laufe der Zeit der plaggengedüngte Acker erhöhte, d. h. der oberste Bodenhorizont, der Plaggenhorizont, wurde mächtiger. Die bisher beobachtete Mächtigkeit des Plaggenhorizontes liegt meistens zwischen 40 und $80 \mathrm{~cm}$.

Sind ausschließlich oder weitgehend Heideplaggen verwendet worden, so ist eine schwärzlichgraue Plaggenauflage entstanden, wogegen die lehmig-sandigen Grasplaggen einen braunen Plaggenesch entstehen ließen. Hier und da sind beide Plaggenarten am Plaggenesch beteiligt, wodurch die Farbe braungrau oder graubraun wurde.

Der Plaggendung wurde fast immer zur Verbesserung von Sandböden verwendet, deren bodentypologische Entwicklung meistens durch Podsol, saure Braunerde und Gley sowie Übergänge zwischen diesen Bodentypen (z. B. Gley-Podsol und Podsol-Gley) repräsentiert sind. In einigen Fällen wurde als ehemaliger Bodentyp die Parabraunerde, der Pseudogley und die Rendzina gefunden; indessen sind dies Ausnahmen. Der ursprüngliche Bodentyp bestimmt um so mehr den Standort, je geringmächtiger die Plaggenauflage ist. Bei einer Mächtigkeit der Plaggenauflage von $80 \mathrm{~cm}$ wird von dieser im wesentlichen der Standort bestimmt, allerdings kann ein untenliegender Gley mit hohem Grundwasser oder eine untenliegende lehmige Bodenart noch einen beachtlichen Einfluß auf den Pflanzenstandort ausüben.

Während der graue Plaggenesch aus Heideplaggen stets den gleichen anthropogenen $\mathrm{A}_{\mathrm{h}}$-Horizont zeigt, ist dies beim braunen Plaggenesch nicht immer der Fall. Es gibt bei letzterem Profile, die den Verdacht nahelegen, daß der anthropogene Horizont nicht etwa nach und nach durch mineralreiche Dungmasse aufgebaut wurde, sondern eine $50-80 \mathrm{~cm}$ mächtige Schicht einer braunen, lehmig-sandigen Bodenart mit einem Male aufgebracht wurde, um einen armen Podsol aus Sand gründlich zu verbessern. Das scheint eine Meliorationsart in den Flottsandgebieten Nordwestdeutschlands zu sein. Eine Beobachtung bei Bersenbrück im südlichen Oldenburg legt diese Vermutung nahe. Mit dieser Art der Bodenverbesserung müssen wir rechnen, wenn wir es mit dem braunen Plaggenesch zu tun haben.

Am bekanntest ist das Profil des Plaggeneschs mit einer grauen Plaggenauflage über einem Podsol. Um die Vorstellung zu erleichtern, wird ein Profilbild dieses Bodentyps (Abb. 1) beigegeben und kurz beschrieben.

$A_{p 1} \quad 0-30 \mathrm{~cm} \quad$ bräunlichgrauer, mittel humoser Sand, Bröckel- und Einzelkorngefüge.

Ap2 $30-70 \mathrm{~cm}$ schwärzlichgrauer, stark humoser Sand, schwaches Bröckelgefüge und Einzelkorngefüge, locker.

Hier beginnt der Podsol.

$\mathrm{A}_{\mathrm{h}} \quad 70-80 \mathrm{~cm}$ dunkelschwarzgrauer, mittel humoser Sand, Bröckel- und Einzelkorn-

$\mathrm{A}_{\mathrm{e}} \quad 80-90 \mathrm{~cm} \quad \begin{aligned} & \text { gefüge. } \\ & \text { hellgrauer Sand, Einzelkorngefüge. }\end{aligned}$

$\mathrm{B}_{\mathrm{sh}} \quad 90-105 \mathrm{~cm}$ braunschwarze, mürbe Eisenhumusorterde, Hüllengefüge.

$\mathrm{B}_{\mathrm{S}} \quad 105-150 \mathrm{~cm}$ rostbrauner (oben dunkler, nach unten heller werdend) Sand, Einzelkorngefüge.

C $\quad$ ab $150 \mathrm{~cm}$ gelblichgrauer Sand, Einzelkorngefüge.

Die Eigenschaften des grauen Plaggeneschs sind: geringe Wasserkapazität, gut durchlässig für Wasser und Luft, locker, gut durchwurzelbar, leicht bearbeitbar, stark saure Re- 


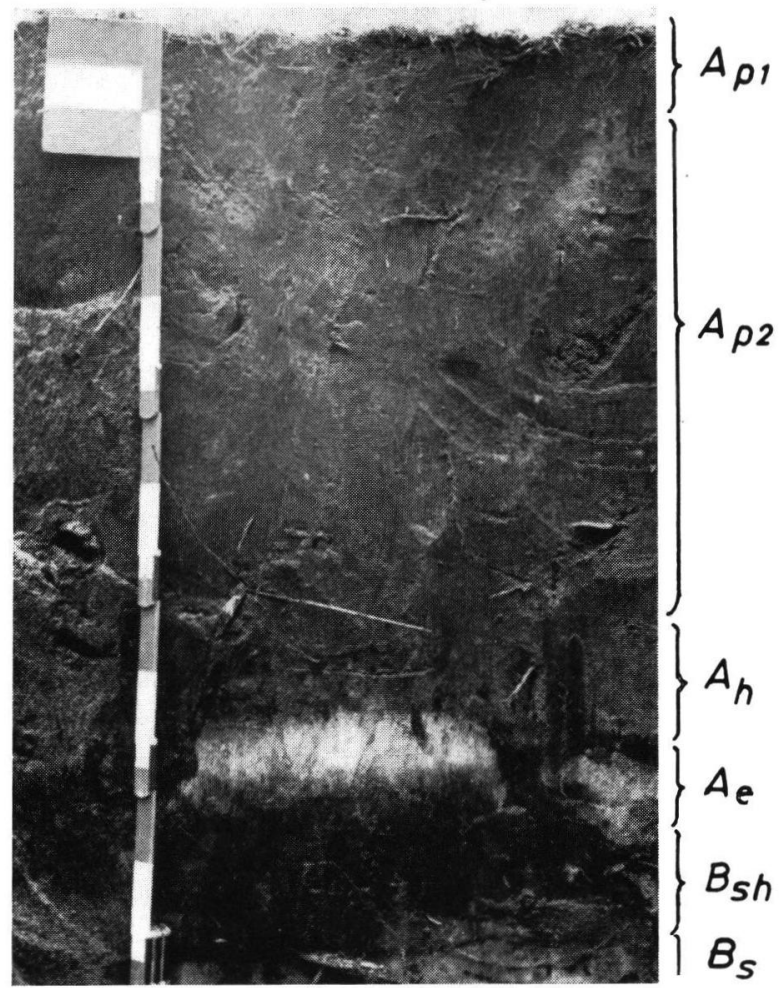

Abb. 1. Grauer Plaggenesch über Gley-Podsol aus fluvioglazialem Sand (Riß). Ort: $2 \mathrm{~km}$ östlich Veldhausen, Grafschaft Bentheim, Nordwestdeutschland.

aktion, niedriger V-Wert, geringe Reserven an Basen, Phosphor, Stickstoff und Spurenelementen. Durch Kalkung und Düngung in den letzten Jahrzehnten ist der Humusgehalt im oberen Horizont etwas gesunken, aber die Basen- und Nährstoffvorräte sind erhöht worden. Der braune Plaggenesch besitzt insgesamt bessere Eigenschaften, besonders dann, wenn die Plaggenauflage lehmig-sandig oder gar sandig-lehmig ist.

\section{Das Alter des Plaggeneschs}

Die Plaggendüngung wurde bis zur allgemeinen Anwendung der Handelsdünger, bis zur letzten Jahrhundertwende, betrieben. Im Emsland wurde vor 20 Jahren noch ein Plaggen-Komposthaufen beobachtet. Das Ende der Plaggendüngung ist sicher feststellbar, indessen ist es sehr schwer, über deren Beginn etwas Genaues zu sagen. Man darf wohl davon ausgehen, daß sie in ihrem Verbreitungsraum von Nordflandern bis Jütland nicht gleichzeitig aufkam, daß sie sich vielmehr von einem Ursprungsraum, vielleicht auch von mehreren, aus verbreitete. Darüber wissen wir nichts. Vielleicht ließe sich dies durch eine systematische Altersbestimmung im ganzen Verbreitungsraum ermitteln, jedoch erforderte das ein relativ enges Netz von Untersuchungen.

Eine Urkunde über die Plaggendüngung liegt im Stadtarchiv von Kleve/N.-Rhein vor. Diese Urkunde bezeugt die Plaggendüngung in der 2. Hälfte des 15. Jahrhunderts; das wären rund 500 Jahre. Aber die Urkunde läßt nicht erkennen, wie lange schon vorher die Plaggenwirtschaft betrieben wurde. Wir haben auf den sandigen Böden des Kreises Kleve und auch noch südlich davon Plaggenesche gefunden. Darüber hinaus gibt es mehrere Urkunden im Verbreitungsraum des Plaggeneschs, indessen reichen diese nicht so weit zurück 
wie der Beginn der Plaggenwirtschaft (G. Niemeier und W. Taschenmacher 1939). Andere Versuche, das Alter der Plaggenwirtschaft zu bestimmen, wurden unternommen, aber die Ergebnisse sind unsicher. Aus dem Alter der Ortsnamen hat man abgeleitet, daß die Plaggendüngung etwa um $800 \mathrm{n}$. Chr. begonnen hat. Die Ableitung wird angezweifelt. Dann wurde der Versuch gemacht, das Alter der Plaggendüngung mit Hilfe von Scherben und anderen Funden zu datieren. Für das Münsterland hat G. NiEmeIER (1939) wahrscheinlich machen können, daß mit der Plaggendüngung hier im Mittelalter, etwa im 10. Jahrhundert oder später begonnen wurde. Aus der Entwicklung der Ackerwirtschaft lassen sich auch Rückschlüsse auf ein etwa gleiches Alter ableiten.

Ein gutes Verfahren für die Altersbestimmung des Plaggeneschs scheint in der ${ }^{14} \mathrm{C}$ Methode gefunden zu sein. Sie besteht in der Bestimmung der Restaktivität des im $\mathrm{Da}-$ tierungsmaterial vorhandenen natürlichen Radiokohlenstoffs. Durch Vergleich der gemessenen Aktivitäten von Datierungsprobe und modernem Kohlenstoff (Oxalsäure vom National Bureau of Standards Washington) ergibt sich der Prozentanteil noch vorhandener Aktivität, der über die Kenntnis der Halbwertszeit des ${ }^{14} \mathrm{C}$ in die bereits verflossene Zeit des Zerfalls, d. h. in das Alter der C-haltigen Probe übersetzt werden kann. Hierbei besteht aber die nicht auszuschließende Schwierigkeit, daß in jedem Jahr im Bodenprofil neue organische Wurzelmasse, im obersten Horizont auch oberirdische Pflanzenmasse, mit modernem ${ }^{14} \mathrm{C}$-Gehalt in den Boden gelangt. Bestimmt man das Alter der organischen Masse eines Bodenprofiles mit Hilfe der ${ }^{14} \mathrm{C}$-Methode, so erhält man folgerichtig ein mittleres Alter der organischen Bestandteile, die von Beginn der Bildung organischer Substanz in dem betreffenden Boden bzw. Horizont bis heute gebildet worden ist. Das ist die Schwierigkeit! Handelt es sich um mächtige humose Horizonte, so wird man in deren tieferem Bereich weniger jüngere und mehr ältere organische Masse erwarten dürfen. In $\operatorname{dem} A_{h}$-Horizont eines Podsols unter einer mächtigen Plaggenauflage werden wir auch mehr, aber nicht nur, ältere organische Masse vorfinden. Ganz frei von junger organischer Substanz sind nur tief begrabene $\mathrm{A}_{\mathrm{h}}$-Horizonte, die nicht mehr von Pflanzenwurzeln erreicht werden. Die Befunde im Gelände müssen mithin bei der Interpretation der ${ }^{14} \mathrm{C}$ Werte berücksichtigt werden.

Eine weitere Sache muß Beachtung finden. Wenn man die organische Substanz des Bodens in Humusstoffgruppen (Fulvosäuren, Braun- und Grauhuminsäuren, Humine) fraktioniert, so ist zu erwarten, daß die höher polymerisierten Humusstoffe ein etwas höheres Alter besitzen als die niedriger polymerisierten, z. B. Fulvosäuren. Daraus muß man ableiten, daß der jeweils bestimmte Alterswert bei den Böden mit viel Grauhuminsäure, Huminen und organo-mineralischer Substanz (z. B. Schwarzerde) höher liegt als bei gleichalterigen Böden mit weniger hochpolymerisierten Humusstoffen, z. B. den Sauren Braunerden und Podsolen. Indessen muß beachtet werden, daß bei starker Versauerung der Abbau der organischen Masse sehr gehemmt sein kann und sich deshalb auch wenig zersetzte Masse lange im Boden erhalten kann. Das trifft für die Podsole zu und auch für den grauen Plaggenesch, der vielfach Podsolhumus enthält. Natürlich wurde durch die Beackerung der Plaggenauflage die Zersetzung der organischen Masse beschleunigt.

Zur Probevorbereitung wird in unserem Labor prinzipiell nur die aufgeschlämmte Ton- und Feinschlufffraktion verwendet, um lediglich die feine organische Substanz und die in organo-mineralischer Bindung vorliegende zu erfassen. Auf jeden Fall werden Wurzeln und deren Fragmente ausgeschieden. Dieses Vorgehen ist notwendig, um junge organische Substanz auszuschließen und eine gute Vergleichbarkeit der Meßergebnisse zu gewährleisten.

Bereits 1959 hat Niemeier das mit der ${ }^{14} \mathrm{C}$-Methode ermittelte Alter von Plaggeneschen bekanntgegeben. Seine Daten geben als Beginn der Plaggendüngung die Zeit um Christi Geburt und noch früher an. Für diese Altersbestimmung wurde Holzkohle ver- 
wendet, die aus dem untersten Teil der Plaggenauflage gesammelt wurde. Möglicherweise war der untere Teil der Plaggenauflage mit dem $\mathrm{A}_{\mathrm{h}}$-Horizont des ursprünglichen Bodentyps gemischt, und dieser $\mathrm{A}_{\mathrm{h}}$-Horizont könnte Holzkohle einer älteren Brandrodung enthalten haben. So könnte sich das relativ hohe Alter gegenüber den neucren Daten erklären. Zwar hat der Autor auffällige Anhäufungen von Holzkohle, möglicherweise herrührend von der Brandrodung, ausgeschlossen, indessen kann aber doch ältere Holzkohle von der Brandrodung in den untersten Teil der Plaggenauflage durch Bodenbearbeitung eingemischt worden sein. Trotz aller Sorgfalt, die der Autor hat walten lassen, ist diese Möglichkeit kaum auszuschließen.

Im Jahre 1962 veröffentlichten FASTABEND und v. RAUPACH die Altersdaten von zwei Plaggeneschen des Emslandes, und zwar wurde der untere Teil der Plaggenauflage untersucht, der mit dem Beginn der Plaggendüngung korreliert. Die mitgeteilten Daten besagen, daß mit der Plaggendüngung etwa 600-800 nach Chr. begonnen wurde, also ein Alter von rund 1150-1350 Jahren anzunehmen ist.

Kürzlich haben Scharpenseel, Tamers und Pietig (1968) die in unserem Institut gemachten Datierungen von 8 Plaggeneschen bekanntgegeben. Von 5 Böden wurde in $60-70 \mathrm{~cm}$ Tiefe eine Probe entnommen und untersucht. Es ergaben sich folgende Altersdaten:

1. Grauer Plaggenesch auf dem Albachtenesch bei Greven/Westf.: $1300 \pm 80$.

2. Grauer Plaggenesch auf dem Marktesch bei Greven/Westf.: $1235 \pm 80$.

3. Grauer Plaggenesch bei Greven/Westf., neben der Hauptstraße, Richtung Schmedehausen: $980 \pm 80$.

4. Grauer Plaggenesch bei Schmedehausen in Richtung Ladbergen, in der Nähe von Greven/ Westf.: $980 \pm 80$.

5. Grauer Plaggenesch in der Kroner Heide bei Greven/Westf.: $1030 \pm 90$.

Von drei weiteren in unserem Institut durchgeführten Altersbestimmungen von Plaggeneschen sind von den oben genannten Autoren mehrere Proben in $10 \mathrm{~cm}$ Abstand untersucht worden. Es sind folgende:

6. Grauer Plaggenesch auf dem Albachtenesch bei Greven/Westf.:

\begin{tabular}{lllr} 
Tiefe & \multicolumn{3}{c}{ Tiefe } \\
$10-20 \mathrm{~cm}$ & $580 \pm 50$ & $40-50 \mathrm{~cm}$ & $790 \pm 60$ \\
$20-30 \mathrm{~cm}$ & $990 \pm 60$ & $50-60 \mathrm{~cm}$ & $730 \pm 80$ \\
$30-40 \mathrm{~cm}$ & $710 \pm 50$ & $60-70 \mathrm{~cm}$ & $1220 \pm 80$
\end{tabular}

7. Brauner Plaggenesch an der Straße Greven-Rheine/Westf., $6 \mathrm{~km}$ vor Rheine:

Tiefe

$\begin{array}{lrlr}20-30 \mathrm{~cm} & 660 \pm 60 & 50-60 \mathrm{~cm} & 1020 \pm 60 \\ 30-40 \mathrm{~cm} & 1170 \pm 60 & 60-70 \mathrm{~cm} & 900 \pm 60 \\ 40-50 \mathrm{~cm} & 1260 \pm 60 & 70-80 \mathrm{~cm} & 810 \pm 60\end{array}$

8. Grauer Plaggenesch an der Straße Lengerich-Iburg, nahe Lengerich/Westf.:

Tiefe

$\begin{array}{lrlr}20-30 \mathrm{~cm} & 860 \pm 60 & 60-70 \mathrm{~cm} & 860 \pm 60 \\ 30-40 \mathrm{~cm} & 910 \pm 60 & 70-80 \mathrm{~cm} & 860 \pm 60 \\ 40-50 \mathrm{~cm} & 1190 \pm 70 & 80-90 \mathrm{~cm} & 3960 \pm 80\end{array}$

$50-60 \mathrm{~cm} \quad 940 \pm 60$

Bei Profil 8. handelt es sich bei der Probe aus $80-90 \mathrm{~cm}$ Tiefe um den $A_{h}$-Horizont des unterlagernden Bodentyps; die organische Substanz dieses ehemaligen Bodentyps hat naturgemäß ein höheres Alter.

Neuere, in unserem Institut durchgeführte Datierungen von 8 Plaggeneschproben, die dankenswerterweise von Dr. H. Mertens und Frau Dr. W. Herbort mit einem der obengenannten Autoren in der weiteren Umgebung von Wiedenbrück/Westf. entnommen wurden, sind in Tabelle 1 aufgeführt. 
Tabelle 1

C-Gehalt und Alter von Plaggeneschproben aus der Umgebung von Wiedenbrück/Westf.

\begin{tabular}{lccr}
\hline Ort & Tiefe in cm & C-Gehalt in $\%$ & Alter \\
\hline 9. 5 km SO Rietberg, Besitztum Speith & $35-45$ & 4,0 & $1200 \pm 70$ \\
10. & $45-55$ & 10,5 & $1140 \pm 70$ \\
11. Brede bei Rietberg, Nähe Friedhof & $40-55$ & 2,0 & $720 \pm 70$ \\
12. Hoffeld, 1,5 km S Rietberg & $55-70$ & 1,4 & $1080 \pm 60$ \\
13. & $70-90$ & 2,0 & $1130 \pm 70$ \\
14. Sinnesche Brede, 3,5 km S Rietberg & $35-55$ & 1,4 & $1540 \pm 60$ \\
15. Am Hohen Lande, 3 km SSO Rietberg & $40-50$ & 2,7 & $900 \pm 90$ \\
16. Auf den langen Stacken, bei Bahnhof Krax, & & & \\
5 km NNO Neuenkirchen & $60-80$ & 1,0 & $1020 \pm 80$
\end{tabular}

\section{Diskussion der Datierungen}

Gesucht wird der Beginn, also das Alter der Plaggendüngung. Die ermittelten Altersdaten von Plaggeneschen sind nicht korrigiert. Unumwunden muß zugegeben werden, daß die Altersdaten von Plaggeneschen mit Fehlern behaftet sind. Vor allem sind die Fehlerquellen in zwei Punkten zu sehen:

1. Wie oben schon ausgeführt, wird in der Plaggenauflage in jedem Jahr neue organische Substanz mit inkorporiertem ${ }^{14} \mathrm{C}$ gebildet. Davon wird jeweils der größte Teil wieder abgebaut, aber ein Teil der organischen Masse geht in den mehr oder minder beständigen Anteil der organischen Bodenmasse ein. Es ist auch möglich, daß die organische Substanz im Bodenprofil nicht streng örtlich gebunden ist. Sogar in Moorböden gibt es eine vertikale Humusverlagerung. Damit ist auch in der Plaggenauflage bis zu einem gewissen Grad zu rechnen. Ferner ist noch an die Durchmischung zu denken, die Pflanzen und Tiere in bekannter Weise vollbringen. Dieser Mischungsprozeß verbietet es ebenfalls, eine regelmäßige und zuverlässige Alterszunahme im Profil von oben nach unten anzunehmen. Aus diesen Tatsachen ist zu folgern, daß das ermittelte Alter des unteren Bereiches der Plaggenauflage nicht das Alter der ältesten Plaggen sein kann, sondern das mittlere Alter des gesamten, in diesem Bereich inkorporierten ${ }^{14} \mathrm{C}$. SCHARPENSeel, Tamers und Pietig (1968) haben den Anteil jüngerer organischer Substanz in tieferen Schichten an einem Modell zu eliminieren versucht, indem sie die Verdünnung der alten organischen Substanz durch jüngere unter Annahme einfachster Verhältnisse überschlagsmäßig berechneten. Danach müßte man bei einem gemessenen Alter von 5000 Jahren 1000 Jahre addieren und bei 3500 Jahren 500 Jahre. Ein Plaggenesch mit einem ermittelten Alter von 1000 Jahren ist nach dieser Berechnung mindestens 100 Jahre älter. Diese Korrektur bringt natürlich nur eine erste Annäherung an das tatsächliche Alter.

2. Ebenso wichtig ist das Alter der verwendeten Plaggen für die Herstellung des Plaggendungs. Diese können ein verschiedenes Alter besitzen, und hierin können erhebliche Fehler begründet sein. Wir müssen davon ausgehen, daß teils alte Plaggen verwendet wurden, teils auch jüngere. Die Plaggen von den regelmäßig geplaggten Heideflächen waren jung, denn diese Plaggen entstammen jungem Heideaufwuchs. Zwar sind solche jungen Heideplaggen viel verwendet worden, jedoch keineswegs ausschließlich. Auch muß bedacht werden, daß die Plaggen von jungem Heideaufwuchs noch ältere Wurzelmasse enthalten können. Ferner sind noch andere Stoffe mit älterer organischer Masse (z. B. Grabenaushub) bisweilen verwendet worden.

Unter Beachtung dieser 2 Punkte muß man die gewonnenen Altersdaten in aller Vorsicht mit einem möglichen Altersspielraum im Hinblick auf den Beginn der Plaggendündung interpretieren. Die Schwankungen des Alters der verschiedenen Schichten in den 
Plaggenböden 7. und 8., vor allem das niedrigere Alter der tieferen Schichten, können mit verschieden altem Plaggenmaterial erklärt werden.

Das hohe Alter der Schicht aus $80-90 \mathrm{~cm}$ Tiefe des Profils 8. ist leicht erklärlich; es handelt sich nicht um die Plaggenauflage, sondern um den $\mathrm{A}_{\mathrm{h}}$-Horizont des unterlagernden Bodens. Uber einen ähnlich gelagerten Fall berichten FASTABEND und v. RAUPACH (1962). Sie ermittelten das Alter von organischer Substanz einer Grabenfüllung unter einem Plaggenesch mit $2480 \pm 120$ Jahren, also ein Alter, das wesentlich höher liegt als die Daten der bisher untersuchten Plaggenauflagen. Niemeier (1959) gibt Altersdaten von rund 2000 Jahren und mehr von Plaggenauflagen bekannt. Diese Daten wurden aus Holzkohle, die in diesem Horizont gesammelt wurde, gewonnen, wie bereits oben gesagt wurde. Diese Holzkohle kann, wenigstens z. T., aus einer älteren Brandrodung stammen, wodurch sich das höhere Alter erklären ließe.

Wenn wir alle Ergebnisse zusammenfassend betrachten und für die möglichen Fehlerquellen einen hinreichenden Spielraum geben, so dürfte als Beginn der Plaggendüngung ein Zeitraum von etwa 800 bis 1200 Jahren vor heute angesetzt werden. Das stimmt einigermaßen mit der Altersbestimmung mit Hilfe anderer Methoden überein.

\section{Literatur}

Fastabend, H. \& F. v. Raupach: Ergebnisse der 14C-Untersuchung an einigen Plaggenböden des Emslandes. Geol. Jb. 79, 863-865, Hannover 1962.

Mückenhausen, E., in Zusammenarbeit mit F. Heinrich, W. Laatsch \& F. Vogel: Entstehung, Eigenschaften und Systematik der Böden der Bundesrepublik Deutschland. DLG-Verlag, Frankfurt/M. 1962.

Niemeier, G.: Die Altersbestimmung der Plaggenböden als kulturgeographisches Problem. Geographischer Anzeiger, Jg. 1939, H. 9/10, Gotha 1939.

Niemeier, G. \& W. Taschenmacher: Plaggenböden. Westf. Forschungen, Mitt. Provinzialinstitut f. westf. Landes- u. Volkskunde 2, Münster 1939.

Niemeier, G.: Von Plaggen und Plaggenböden. Jb. d. emsländischen Heimatvereins 3, Meppen 1955. - - 14C-Datierungen der Kulturlandschaftsgeschichte Nordwestdeutschlands. Abh. d. Braunschw. Wissenschaftl. Gesellschaft 11, Braunschweig 1959.

Scharpenseel, H. W., M. A. Tamers \& F. Pietig: Altersbestimmung von Böden durch die Radiokohlenstoff-Datierungsmethode. I. Methoden und vorhandene ${ }^{14} \mathrm{C}-\mathrm{Daten}$. II. Eigene Datierungen. Zeitschr. f. Pflanzenern., Düngung, Bodenkunde, 1968 (im Druck).

Wohlrab, B. \& Chr. Langner: Uber den Wasserhaushalt verschiedener Plaggenesche. Zeitschr. f. Pflanzenern., Düngung, Bodenkunde 109, 3, 227-239, 1965.

Manuskr. eingeg. 25. 5. 1968.

Anschrift der Verf.: Prof. Dr. Dr. E. Mückenhausen, Prof. Dr. H. W. Scharpenseel und Dipl.Chem. F. Pietig, Institut für Bodenkunde der Universität Bonn, 53 Bonn, Nußallee 13. 